\title{
Embodied Experiences Associated with Obesity and the Management of Bodyweight. Gender and Social Differences
}

\author{
Louise H. Smith ${ }^{1} \&$ Lotte Holm ${ }^{1}$ \\ ${ }^{1}$ Department of Human Nutrition, University of Copenhagen, Denmark \\ Correspondence: Louise H. Smith, National Research Center for the Working Environment, Copenhagen, \\ Denmark. Tel: 45-3916-5291. E-mail: 1hs@arbejdsmiljoforskning.dk
}

Received: June 6, 2012 Accepted: July 24, 2012 Online Published: September 5, 2012

doi:10.5539/jfr.v1n4p7

URL: http://dx.doi.org/10.5539/jfr.v1n4p7

\begin{abstract}
In many affluent Western societies the less educated are at higher risk of developing obesity. Within a conceptual framework of sociology of embodiment, this study analyzed the embodied experiences associated with obesity and the management of body weight among women and men with different social backgrounds. Qualitative in depth interviews were conducted with 20 Danish middle-aged men and women who were categorized as clinically obese in a national dietary survey. The study found a devastating impact of obesity in the lives of highly educated women related to motherhood, career and wifehood which interrelated with persistent efforts to lose weight and repeated involvement in a great variety of weight-loss activities. In contrast, body weight among the less educated interviewees was a concern only in specific situations of everyday life, and it was a health-related concern especially for those men who had experienced weight-related disease, which interrelated with less commitment to, and variation in, weight-loss activities. These findings may help to explain why obesity is least prevalent among highly educated women in Danish society as well as other western societies. A marked difference between men and women was that only few men followed dietary regimes which involved a focus on cooking or changing eating habits on their own initiative. In addition they did not participate in commercial weight-loss programs. We discuss how the social and gendered differences found relate to wider societal contexts and how the findings may both challenge and inform public health promotion.
\end{abstract}

Keywords: obesity, embodied experiences, weight management, social class, qualitative study

\section{Introduction}

The increasing prevalence of obesity is a major public health concern. Obesity occurs in all social groups, but in many affluent Western societies there is an unequal social distribution of obesity - in Denmark, most markedly, according to educational background (Heitmann, 1991; Heitmann, 2000a; McLaren, 2007). In a recent study obesity was significantly associated with low education especially for women. Prevalence of obesity was the lowest among women with medium or long higher education (Groth M. V et al., 2009).

In the medical discourse on obesity the consequences of obesity for physical health, along with problems arising from the social stigmatization of obese individuals, are prominent concerns (WHO, 1997; Krauss et al., 2000; Blaine et al., 2007; Rogge et al., 2004) . The question is, however, which concerns exist among those who experience clinically defined obesity themselves, and how do differences in experiences reflect the prevalence of obesity in different social groups and genders?

In Denmark public health policy on obesity is based on a liberal political ideology, focusing on individual responsibility and on recommendations of healthy lifestyles (Vallgårda, 2007). Thus, a core element in health promotion has been the advocacy of guidelines for healthy eating, traditionally in the form of a list of seven dietary recommendations (such as to eat less fat, less sugar, more fruit, more vegetable, more fish, more fibre, maintain body weight within a recommended range, and so on). In the latest revision of these guidelines, an eighth recommendation has been added: "Maintain a level of physical activity of a minimum of 30 minutes per day" (Astrup et al., 2005). Thus, in Denmark, the fight against obesity focuses on persuading citizens to adopt recommended dietary practices and increase their physical activity.

So far, this policy has not diminished the social inequality in obesity. 
Research has shown that obese individuals are dissatisfied with their body-shape, and that being overweight affects self-esteem (Grignard et al, 2000; Newmark-Sztainer et al., 1999). However, research suggests that concerns about being overweight are not uniform or evenly spread throughout populations. In contemporary Western societies a slim body ideal applies more to women than to men (Bordo, 1993), and it has been demonstrated that the social costs of obesity, measured by education, employment and income, are especially profound for women (Gimlin, 2007). Furthermore, being overweight has been found to have a more negative relationship with self-esteem among high socio-economic status (SES) individuals than among middle- and low-SES individuals (Miller \& Downey, 1999). It is likely that such variations in embodied experiences and concerns about body weight will be reflected in variations in practices associated with weight management. The objective of the current study is to explore whether, and if so how, variations in embodied experiences of overweight are reflected in practices related to weight management.

In what follows we first present the design of the study and the methods used. We then describe the experience of medically defined obesity as reported by men and women from different social backgrounds. We then ask how weight management varies in the studied population. In the discussion we relate these variations to differences in embodied experience, and we discuss how the social and gendered differences found relate to wider societal contexts and how the findings may inform public health promotion.

\section{Method}

The study consisted of qualitative, semi-structured, in-depth interviews. Qualitative methods are suitable for gathering rich descriptions of experiences and promoting inclusive study of the kind that does not confine the investigation to a predetermined set of interviewee-responses (Kvale, 1996). In all 10 Danish women and 10 Danish men from various social backgrounds participated. In contrast with much qualitative research on obesity, interviewees in the current study were not selected through their participation in, or membership of, weight reduction clubs and programs as this would have included only those individuals who were actively trying to lose weight. Instead they were recruited from among the participants in a representative survey on dietary habits in Denmark which included self-rated height and weight (Fagt et al., 2002). It was anticipated that this recruitment would provide a more comprehensive understanding of experiences related to clinical obesity and to weight management, because in this way both those who were involved in the process of losing weight (whether alone or in organized programs) and those who were not would participate.

Criteria for inclusion in the current study were BMI of 30-34 (denoting obesity). Further criteria for inclusion were: age 35-55 years; living in multiple-person household in urban community; and in paid occupation (all criteria to be satisfied at time of participation in the survey). Criteria related to differences in social background were defined according to educational history, because in Danish research this variable most clearly identifies social difference with respect to eating habits and obesity (Groth et al., 2001; Dynesen et al., 2003; Heitmann, 2000) inclusion criteria were either basic or vocational education, or medium or long cycle of higher education. Interviewees were selected to ensure an even distribution of gender and education. Five women and five men with basic education participated together with five women and five men with higher education. Only three individuals refused to participate in the study.

Key themes of the interviews included life history, everyday life, and explanations, experiences and practices concerning health, food, physical activity and personal weight.

Interviews lasted on average 3 hours (range 1.5-4 hours) and were in most cases conducted on two separate occasions. All interviews were audio-taped, transcribed verbatim and thematically coded using the software program Atlas.ti (Computer software, 1999). The interview material was read through several times with the aim of comparing groups defined by gender and educational history.

This article presents findings concerning experiences of being overweight and weight management engaged in through the life-course. For findings concerning explanations of overweight and variations in how recommended healthy lifestyles are integrated in the everyday life in different social classes see articles by L. Smith \& L. Holm $(2010 ; 2011)$. In the following sections, individuals with primary school, high school or vocational education are characterized as 'less educated', and individuals with a university diploma as 'highly educated'.

\subsection{Conceptual Framework}

The conceptual framework for the study was the sociology of embodiment (Turner, 1992).

Jonathan Watson (Watson, 2000) who was the main inspiration, distinguishes between four interdependent dimensions of embodiment when interpreting the way Scottish working-class men experience and make sense of their personal health. The dimensions are: the normative dimension, which categorizes according to features of 
presentation and appearance such as body shape; the experiential dimension, which categorizes the experience of emotion, in the sense of experiencing well-being or not feeling well; the visceral dimension, which relates to hidden biological depths and refers to what can only be medically visualized or be experienced through the experiential dimension for instance in case of illness; and, finally, the pragmatic dimension, which refers to the body's ability to fulfill everyday social obligations and gendered roles (Watson, 2000).

The pragmatic, everyday dimension of embodiment is typically the primary mode of agency in the social world, but it combines with other dimensions of embodiment, depending on context and situation (Watson, 2000).

Watson's concept of embodiment, with its different sub-dimensions was applied in this study in order to capture differences in experiences of obesity.

\section{Results}

Seven interviewees reported weight-loss since participation in the original survey. Of these, four had experienced major weight-loss $(12-30 \mathrm{~kg})$. Six interviewees were in the process of losing weight at the time of the interview, while others were not. All except one, however, reported experiences associated with the intention to lose weight at some point during their life-course; but most had relapsed. This suggests that their body weight had at some point been a cause of concern. However, some important differences in concerns appeared in the interviews. These differences relate to whether, and in what sense, obesity was perceived to be a concern, and to the character of any weight management engaged in through the life-course. In the following, differences in these respects between genders and social groups are highlighted.

\subsection{Variations in Embodied Experiences of Obesity}

\subsubsection{Experiences of the Highly Educated}

For the highly educated women, persistent concerns about body weight appear to penetrate everyday pragmatic embodiment related to motherhood, career and wifehood. An example is Sarah, a 46 year-old unemployed actress who reports that she fears that her weight will have a negative effect on her children's social life. She explains: 'I fear that when they grow older they will be bullied by their friends for having a fat mother - which would be terrible. They already say: 'You are fat'. She also reveals how her weight has influenced her career, she says: 'My agent has told me directly, you have to lose weight to get parts'. Another example is 58 year-old Anne, who, as a young physiotherapist, received adverse comments from colleagues who felt that her weight was inappropriate for someone with her role in the health sector. At that time this caused her great concern.

Experiential dimensions of embodiment were reported to be affected too. Anne, the physiotherapist explains, for example: 'Previously, I weighed more than I do today. I will never do that again, it really reduced my quality of life; I felt I was trapped in fat. Where was I?'

Julia, a 53 year old lecturer, also evoked experiential embodiment both when she reported what motivated her to embark on weight-loss activities and when she explained what motivated her to keep losing weight. To take the first of these, she explains: 'When you are contemplating losing weight the main concern is what others might think of you, and that you are looked down upon. That is what makes you fed up with being overweight'. However, when explaining what motivates her to keep losing weight she also emphasizes experiences with practical aspects. She says: 'What is so rewarding about losing weight is the ease with which you can do things like getting out of bed and walking up the stairs ... It is simply a much easier way of going through life'.

Like the highly educated women, some of the highly educated men had had their weight commented upon in connection with work. Also their weight had been commented upon by female family members. However, obesity did not seem to affect every day pragmatic dimensions of embodiment in the pervasive manner witnessed in highly educated women. Some highly educated men express a high degree of concern about their body weight, but only in relation to health risks; they have commenced a change in lifestyle since participation in the original survey and anticipate losing weight in the future. Others had not changed their lifestyle and offered harsh criticism of the societal discourse on obesity.

Paul, a part-time architect in his late fifties, is one of the highly educated men in whom the experiential dimension associated with possible effects on visceral embodiment is latent. He especially worries that his weight might affect his bone structure, and whether obesity will shorten the length of his life.

Jim, a 45 year-old lawyer, is one of the highly educated men who challenge societal risk discourses on obesity. He perceives society to be subjected to 'hysteria' and 'fat phobia'. He explains his position in this way: 'In my opinion, weight is a ridiculous place to have your focus. I feel well with my body and myself - and I do feel I am fit. I can easily work for many hours every day'. He describes his overweight in terms of carrying a 'career 
stomach'.

Bob is a 60 year-old head teacher. At work, he is continually reminded of societal concerns, especially about childhood obesity. However, like Michael, he is critical of societal discourse on obesity. He accentuates inclusion and open-mindedness, both in his teaching and in opposition to the standardizing norms he finds implicit in societal discourse on obesity.

\subsubsection{Experiences of the Less Educated}

For the less educated participants, everyday embodied practices as workers, mothers, fathers, wives and husbands appear to be less affected by a high BMI. Where the less educated women were concerned, being obese typically affected fewer dimensions of embodiment. Everyday pragmatic embodiment was not affected to the same degree as it was in the case of the higher educated women. Thus, body weight was not reported to be a concern in relation to work, motherhood, partners or close social relations. Rather, the concerns appeared to be related to encounters with strangers.

Helen, who is 42 years old, and has retired early from unskilled work due to illness, explains: 'If anyone has commented (on my weight) they have been total strangers. They believe they have the right to comment if you eat an ice cream or something like that'.

Shopping for clothes is another typical situation in which less educated women report feelings of self-consciousness about their body shape in a negative manner. Susan, who is a 45 year-old helper in a day nursery, explains 'It irritates me extremely that the size is wrong. Whether I look like this or should feel ashamed in front of my children and their friends or my own, this is not a concern'. She explains 'I know them and they know me' implying that appearance is not important in these close relations.

Although intimate social relations appeared not to be negatively affected by normative dimensions of embodiment, concerns about weight could become more important at specific times of life. Thus, Susan reports that, years ago, getting married was a motivational factor in losing weight. Being overweight was not in keeping with her image of a bride, and she lost weight. She regained weight after the wedding, and today she is concerned about her weight only when shopping for clothes.

Most of the less educated men had lost weight since participation in the dietary survey. Some - such as 55 year old Bill, who was unemployed from clerical work and had lost weight because of a dental operation which forced him to change his eating habits - had done so without adopting weight-loss as a specific aim. Others had done so upon receiving medical advice on the development of a weight-related disease.

Some of the less educated men report an experience of weight 'creeping up' on them unnoticed. An example is Jeff, a 42 year-old skilled workman. He was diagnosed as diabetic 2 years ago, and since then he has lost $30 \mathrm{~kg}$. Even though Jeff links his main concern about his body weight today to health, he does report in retrospect how his former weight caused problems with practical activities in everyday life. At the same time, he explains why his attitude to the impact of his being overweight seemed to change after weight-loss. He says: 'It was troublesome to get around and also to work - but it does not suddenly happen from one day to the next. You do not notice that you have become more and more heavy. It creeps up on you'. Later he continues: 'It is not until you have lost weight again that you realize how troublesome it was being larger'. Neither his wife nor others in his circle of acquaintances had commented on his weight gain, for, as he says, 'again, it does not happen from one day to the next'.

Concerns connected with the visceral dimension of embodiment were shared by both higher and less educated interviewees. However, whereas the higher educated participants refer to this in terms of future risk of disease, the less educated - and especially the less educated men - refer to visceral dimensions based on experience: when being diagnosed with weight-related disease.

\subsection{Weight Management through the Life-course}

Commitment to, and strategies for, managing personal body weight differed markedly between the genders, but to some degree also according to social background. The differences relate to variations in weight-loss activities and commitment to weight loss. An overview of the weight-loss activities interviewees had engaged in according to gender and educational background is given in Tables 1 and 2 .

The activities here are divided into three categories: Private weight-loss activities (i.e. changes in daily habits which were taken up by the interviewees on their own initiative). Program participation (i. e. activities taken up with the encouragement of a commercial or public program such as a dieting or slimming club). This category of activities also includes counseling and psychotherapy. The third category, medically prescribed treatment, refers 
to activities taken up which are initiated, and sometimes supervised, by a GP or hospital.

Table 1. Weight-loss activities engaged in through the life-course: women

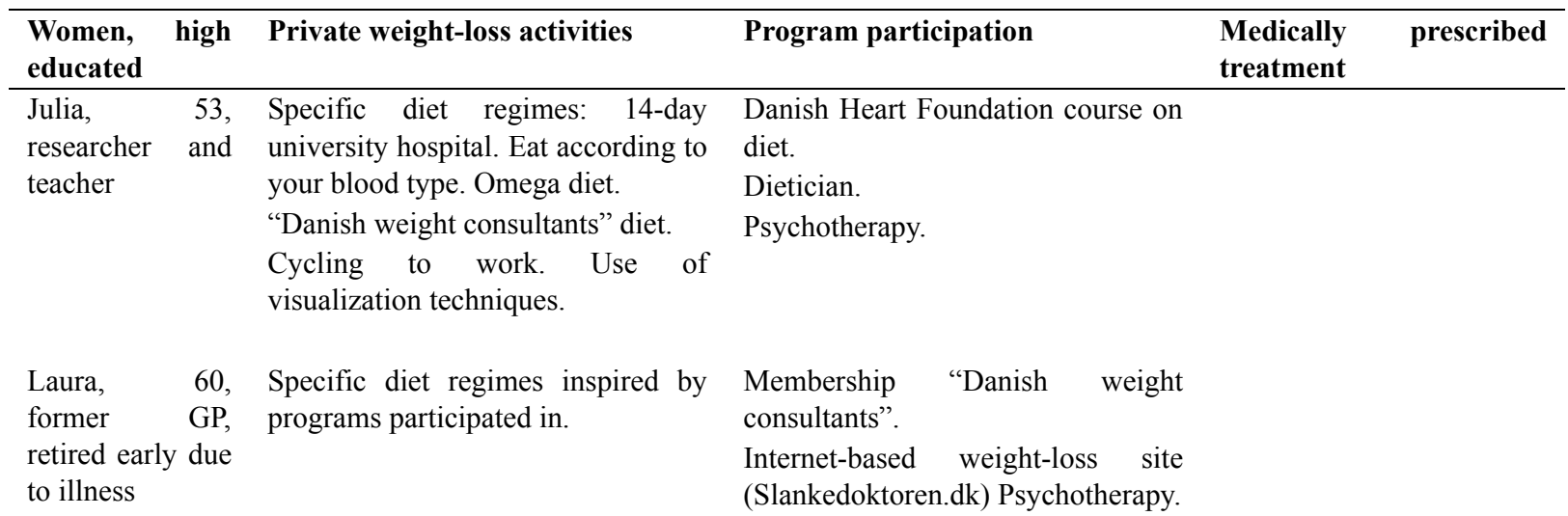

Anne, 58, Specific diet regimes inspired by physiotherapist programs participated in. Walking.

Sarah, 46, Specific diet regimes inspired by unemployed programs participated in.

actress Swimming.
Diet therapy. Gym group.

Colonic therapy.

"Danish weight consultants".

Weight-loss course in Fitness center.

Weight-loss course with coach (DGI).

Internet-based weight-loss site (Slankedoktoren.dk.)

Diet coach.

Psychotherapy.

Fitness center with personal trainer. Weight-loss medication Psychotherapy.

Weight-loss medication
42, Specific diet regimes inspire

Danish Weight consultants and Herba-life.

Avoiding sweet stuff and exercise.

\section{Women, lower \\ educated}

Belinda, 50,

shop assistant

Mary, 52, Follow principles of "the healthy factory worker food pyramid" from supermarket booklet.
Local "healthy city" dietary advice project.

"Danish weight consultants".

Dietician through random participation in hospital research project.

\footnotetext{
Helen, 42, Eat less fat and sugar.

former unskilled

worker, early

retired due to

illness

Susan, 45, Specific diet regime from GP in

helper in a day connection with weight-loss medication.

Clare, 60, Specific diet regime inspired by clerical worker Atkins Diet (no starch and sugar).
}

Weight-loss medication. 
Among the highly educated women the pervasive effect of being overweight on embodiment interrelates with weight-loss being a desired and continuously sought-after goal. It is characteristic of these women to report that they have been more or less constantly engaged in trying to lose weight for years.

The weight-loss activities these women have engaged in through their life-course include following a variety of dietary regimes the character of which varies according to popularity at the time. All the highly educated women had participated in commercial weight-loss programs as well. In addition, most of the highly educated women interviewed had participated in psychotherapy. This was typically for reasons other than reducing their body weight, but weight concerns had nevertheless been a theme in the therapy. In the study only one highly educated woman had not consulted a psychologist. Activities in this group also included taking prescribed weight-loss medication.

Like the highly educated women, the less educated women had engaged in personal weight-loss activities like dieting. However, the less educated women much more rarely participate in weight-loss programs, and they do not participate in psychotherapy. Reports on weight-loss activities from these women show that they are less persistent in their efforts than the higher educated women: they report that for longer periods they ignored their overweight. Belinda, a 50 year old shop assistant, has in recent years participated in a number of healthy eating and weight-loss programs which sets her apart from the other women with less education. Her weight-loss activities include joining a course on healthy cooking and eating - which, as she points out, was financed by the local community and thus charged low fees. This was important for her at the time. She later joined a commercial weight-loss program. The cost of weight-loss programs was also a concern for Mary, a 42 year old factory worker who recounts that she had not engaged in weight-loss activities before she was diagnosed with insulin resistance and obtained a prescription for free dietary advice. She revealed that she would never spend money on a membership of a slimming organization.

The interviews reveal that, regardless of educational history, only few men followed dietary regimes on their own initiative. None of the men participated in commercial weight-loss programs. Instead they made various modifications to their daily habits, such as cutting down on sweet foods and doing more physical exercise. Program participation is typically not seen as an option. The highly educated Michael's reply to a question about this illustrates the point: 'Forget it, that kind of organized stuff ... Weight Watchers etc ... that is something women have created for women. It is an extension of women's magazines'.

Often, it is a wife or female partner who urges the man to begin a weight-loss activity. Paul, who had just been placed on the new Danish health service 'Physical Exercise on Prescription' by his GP, explained that his wife had read about this new treatment program and had made him go to the doctor. It is noteworthy that it is especially the less educated men who are prescribed weight-loss treatment by their GP upon developing weight-related diseases. Examples here are Jeff, the 42 year-old skilled workman, and George, a 55 year-old unskilled workman. Both were prescribed treatment by dietician upon being diagnosed with diabetes and heart disease, respectively. 
Table 2. Weight-loss activities engaged in through the life-course: men

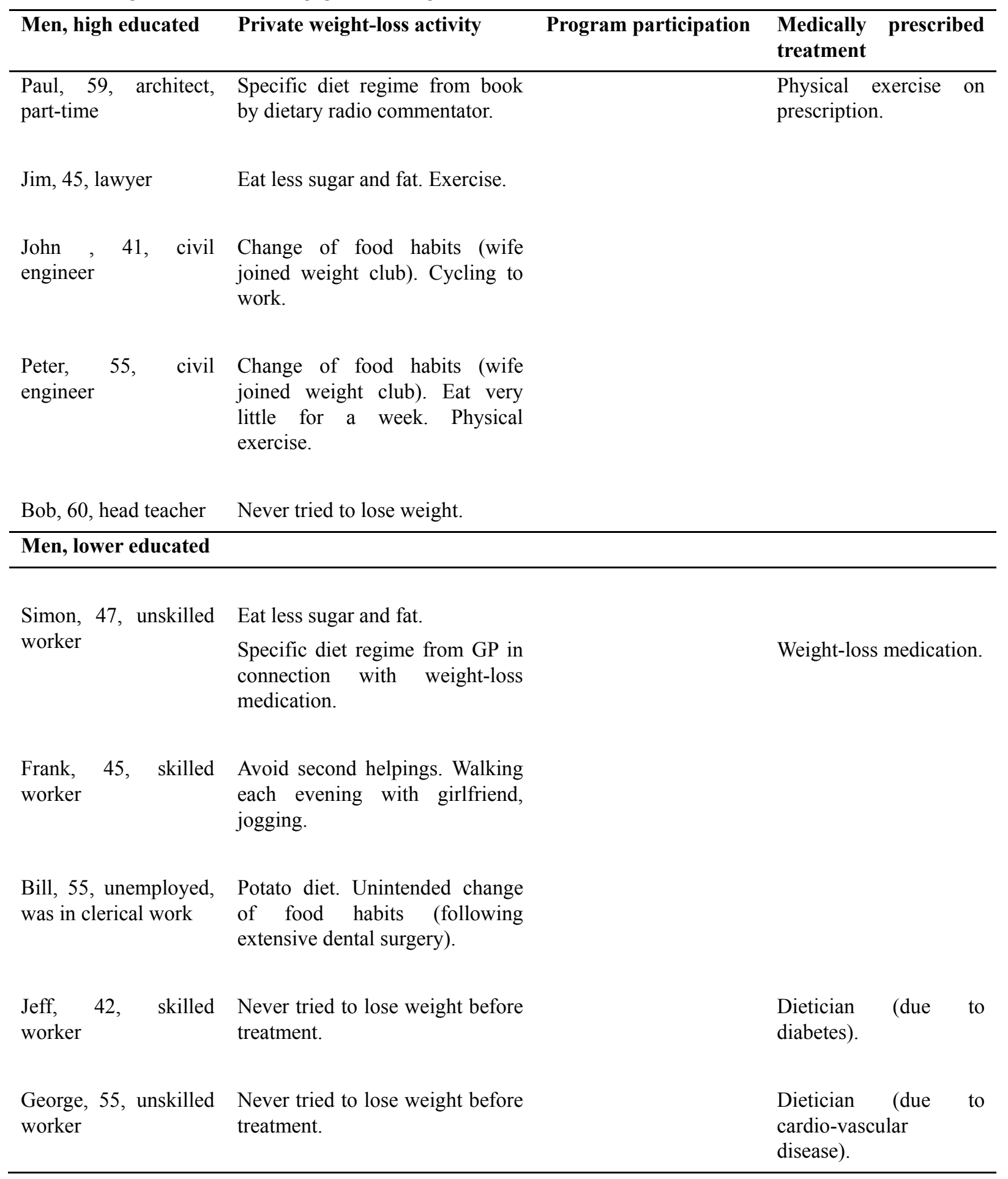

\subsection{Experience and Management of Obesity}

In summary the study shows that the pervasive concerns about obesity and overweight which were found among the highly educated women interrelate with persistent efforts to lose weight and continuous engagement in a great variety of weight-loss activities. By contrast, the more limited concerns not influencing everyday embodiment which were found among the men and among the less educated women correlate with much less weight-loss activity. In these latter cases, weight-loss activities were often reactive following the embodied experience of weight-related ill-health. However, differences in weight-loss activities were not related to differing embodied experiences alone. In the interviews with individuals - especially women - with less 
education, references to material resources indicate that the cost of participation in organized slimming activities was a barrier to losing weight for these individuals.

\section{Conclusion and Discussion}

In this study we have analyzed gender and social background differences related to the experience of clinical obesity and weight management through the life-course.

The study places the diverse impacts of obesity on differing population groups in the social and cultural contexts of everyday life for men and women with different social backgrounds. In highlighting variations in which dimensions of embodiment were affected, and to what degree, Watson's differentiated concepts helped us to focus on the way embodied experience relates to social obligations, performance in everyday life, normative body ideals, and personal experience of wellbeing (Watson, 2000).

For the highly educated, and especially the higher educated women in the study, the experience of being overweight penetrates all dimensions of embodiment, including everyday pragmatic embodiment, both in relation to work and to family life. In Watson's terms, these women do not seem to have any dimension of embodiment to move to which is not affected negatively by obesity. This is not the case, at any rate to the same extent, for men and for women with less education. Here being overweight appears, for the most part, to raise concerns only in relation to health and visceral embodiment. The study therefore suggests that for some individuals obesity might not be a concern if it is not presently perceived as a threat to health, or to visceral embodiment. This finding is in accord with the findings of a study of teenagers in working-class communities, that found neither fear of fatness nor any connection between being overweight or obese and body dissatisfaction (Wills et al., 2006); and a study of men's understanding of appropriate weight-for-height, where it was shown that the men found the clinically defined 'healthy' BMI (body mass index) standard much too strict (Monaghan, 2007).

Turning to weight-loss activities, the study found a relation between concerns about body weight and efforts to lose weight. The all-encompassing influence of obesity in the lives of the highly educated women interrelates with persistent efforts to lose weight and repeated involvement in a great variety of weight-loss activities, whereas the more limited concerns in other social groups interrelate with less commitment to, and variation in, weight-loss activities.

This may help to explain why the prevalence of obesity is lowest among highly educated women.

A marked difference between men and women was found, in that men did not adopt weight-loss activities which involved a focus on cooking or changing eating habits on their own initiative. This might be ascribed to the fact that such activities, despite changes in gender relations in recent decades, are seen to be more appropriate for women (Jensen \& Holm, 1999). It has been suggested that overweight men are therefore caught in a trap: they do not fit the masculine ideal of muscle tone and fitness (Bordo, 1999), nor can they change this through participation in dietary weight-loss activities without further compromising their male identity (Watson, 2000). The perception, found in this study, that slimming groups are an extension of women's magazines, and the fact that wives and girlfriends acted as important intermediaries and partners in the men's weight-loss activities, support this interpretation. The reference, by women with less education, to the cost of organized weight-loss activities, shows that factors other than embodied experience had an important influence on uptake of weight-loss activity.

Besides the small sample size, another potential limitation of the present study is the age composition of the sample. It included only middle-aged individuals and might not, therefore, reflect the experience of younger generations. As gender relations are in a process of change (Bonke, 2009), in future studies it will be particularly important to investigate whether gendered experience and practices differ among younger individuals in the same ways, and to the same extent, as they do in this study. Looking ahead to future research, the study in addition raises the question whether the health consequences of obesity differ from one social group to another, as is the case with other diseases such as cancer (Dalton \& Joachim Schüza, 2008). Our findings suggest that the less educated, and especially less educated men, engage in weight-loss activities mostly upon onset of weight-related disease or diagnosed risk. This might imply that obesity is more likely to lead to ill-health in this group. In the study, the highly educated women report continuous engagement in slimming activities. This could be beneficial for their health if it helps them to maintain healthy eating patterns and to be more physically active and fit. On the other hand, pervasive concern about personal body weight might be harmful for these women's health if it leads to excessive worry or unhealthy slimming practices. These issues need to be addressed.

This study has explored socio-culturally contextualized obesity management from an insider's perspective. It 
highlights the way in which medically defined obesity is embodied, experienced and articulated, and it shows how weight management is embedded in different life histories and daily lives.

The present public health initiatives - foregrounding recommendations on healthy lifestyle and individual responsibility seem to fail to address the social inequality in obesity.

Against this background, the current study proposes that health initiatives aiming to reduce social inequality in obesity should reflect the structural conditions of everyday life, making the healthy choice the easy choice for all. In public health promotion it would seem obviously sensible to initiate dialogues with the population groups most at risk of obesity in order to investigate their ideas and proposals for health promotion initiatives.

Furthermore, the development of weight-loss programmes which appeal to men would likewise seem appropriate, taking the findings of the study in to consideration.

For women cheap and varied weight-loss treatment programmes, including psychological treatments, would be helpful.

When recalling their former problems, those who were currently losing weight with success described troublesome interactions with material things and practical hardships in the performance of ordinary daily activities. We suggest that these retrospective descriptions highlight an important practical aspect of being overweight or obese which is often overlooked. If this is right, public health efforts might benefit by putting more emphasis on the everyday practical constraints of obesity, rather than arguing mainly on the basis of health risk and social stigmatization.

\section{Acknowledgements}

The authors sincerely thank the women and men who participated in this study.

\section{Reference}

Astrup, A., Andersen, N. L., Stender, S., \& Trolle, E. (2005). Kostrådene 2005 [DietaryRecommendations, 2005]. Søborg: Ernæringsrådet og Danmarks Fødevareforskning, 1-88

Blaine, B. E., Rodman, J., \& Newman, J. M. (2007). Weight loss treatment and psychological well-being: a review and meta-analysis. Journal of Health Psychology, 12, 66-82. http://dx.doi.org/10.1177/1359105307071741

Bonke, J. (2009). Forceldres brug af tid og penge på deres børn [Parents' use of time and money on children]. Odense: Syddansk Universitets forlag.

Bordo, S. (1993). Unbearable Weight: Feminism, Western Culture, and the Body. Berkley: University of California Press.

Bordo, S. (1999). The Male Body - A New Look at Men in Public and the Private. New York: Farrar, Straus and Giroux.

Computer software. (1999). Atlas/ti. [Computer software] (Version 4.2) [Computer software]. Berlin: Scientific Software Development.

Dalton, S. O., \& Joachim Schüza. (2008). Social inequality in incidence of and survival from cancer in a population-based study in Denmark, 1994-2003: Summary of findings. European Journal of Cancer, 44, 2074-2085. http://dx.doi.org/10.1016/j.ejca.2008.06.018

Dynesen, A. W., Haraldsdóttir, J., Holm, L., \&Astrup, A. (2003). Socio-demographic differences in dietary habits described by food frequency questions - results from Denmark. European Journal of Clinical Nutrition, 57, 1586-1597. http://dx.doi.org/10.1038/sj.ejcn.1601728

Fagt, S., Matthiessen, J., Trolle, E., Lyhne, N., Christensen, T., Hinsch, H. J. (2002). Danskernes kostvaner 2000-2001 [Dietary habits in Denmark 2000-2001]. Søborg: Fødevaredirektoratet.

Gimlin, D. (2007). Constructions of ageing and narrative resistance in a commercial slimming group. Ageing \& Society, 27, 407-424. http://dx.doi.org/10.1017/S0144686X06005757

Grignard, S., et al. (2000). Characteristics of Adolescent Attempts to Manage Overweight. Patient. http://dx.doi.org/10.1016/S0738-3991(02)00217-3

Groth M. V, Fagt, S., Matthiesen, J., \& Biltoft-Jensen, A. (2009). Dimensions of socioeconomic position related to body mass index and obesity among Danish women and men. Scandinavian Journal of Public Health, 37, 418-426. http://dx.doi.org/10.1177/1403494809105284 
Groth, M. V., Fagt, S., \& Brøndsted, L. (2001). Social determinants of dietary habits in Denmark. European Journal of Clinical Nutrition, 55, 959-966. http://dx.doi.org/10.1038/sj.ejen.1601251

Heitmann, B. L. (1991). Body fat in the adult Danish population aged 35-65 years: An epidemiological study. International Journal of Obesity, 15, 535-545.

Heitmann, B. L. (2000). Ten-year trends in overweight and obesity among Danish men and women aged 30-60 years. International Journal of Obesity, 24, 1347-1352. http://dx.doi.org/10.1038/sj.ijo.0801388

Jensen, K. O., \& Holm, L. (1999). Preferences, Quantities and Concerns - Socio-Cultural Perspectives on the Gendered Consumption of Foods. European Journal of Clinical Nutrition, 53, 351-359.

Krauss, R. M., Eckel, R. H., Howard, B., Appel, L. J., Daniels, S. R., Deckelbaum, R. J., et al. (2000). AHA Dietary Guidelines: Revision 2000: A Statement for Healthcare Professionals From the Nutrition Committee of the American Heart Association. Circulation, 102, 2284-2299. http://dx.doi.org/10.1161/01.CIR.102.18.2284

Kvale, S. (1996). InterViews: An Introduction to Qualitative Research Interviewing. Sage Publications.

McLaren, L. (2007). Socioeconomic Status and Obesity. Epidemiologic Reviews. http://dx.doi.org/10.1093/epirev/mxm001

Miller, C. T., \& Downey, K. T. (1999). A Meta-Analysis of Heavyweight and Self-Esteem. Personality and Social Psychology Review, 3, 68-84. http://dx.doi.org/10.1207/s15327957pspr0301_4

Monaghan, L. F. (2007). Body Mass Index, masculinities and moral worth: men's critical understandings of appropriate weight-for-height. Sociology of Health \& Illness, 29, 584-609. http://dx.doi.org/10.1111/j.1467-9566.2007.01007.x

Newmark-Sztainer, D., et al. (1999). Issues of Self-Image among Overweight African-American and Caucasian Adolescent Girls: A Qualitative Study. Journal of Nutrition Education, 31, 311-320. http://dx.doi.org/10.1016/S0022-3182(99)70484-X

Rogge, M. M., Greenwald, M., \& Golden, A. (2004). Obesity, Stigma, and Civilized Oppression. Advances in Nursing Science, 27, 301-315.

Smith, L. H., \& Holm, L. (2010). Social class and body management. A qualitative exploration of differences in perceptions and practices related to health and personal body weight. Appetite, 55, 311-318. http://dx.doi.org/10.1016/j.appet.2010.07.001

Smith, L. H., \& Holm, L. (2011). Obesity in a life-course perspective: An exploration of lay explanations of weight gain. Scandinavian Journal of Public Health, 39, 396-402. http://dx.doi.org/10.1177/1403494810395819

Turner, B. S. (1992). Regulating Bodies.Essays in Medical Sociology. London.

Vallgårda, S. (2007). Health inequalities: Political problematizations in Denmark and Sweden. Critical Public health, 17, 45-56.http://dx.doi.org/10.1080/09581590601071705

Watson, J. (2000). Male Bodies.Health, Culture and Identity. Buckingham: Open University Press.

WHO. (1997). Obesity: preventing and managing the global epidemic. Report of a WHO Consultation on Obesity. Geneva 3-5 June 1997. Geneva: WHO Publications.

Wills, W., Backett-Milburn, K., Gregory, S., \& Lawton, J. (2006). Young teenagers' perceptions of their own and others' bodies: A qualitative study of obese, overweight and 'normal' weight young people in Scotland. Social Science \& Medicine, 62, 396-406. http://dx.doi.org/10.1016/j.socscimed.2005.06.014 